\title{
Length requirements for numerical-relativity waveforms
}

\author{
Mark Hannam, ${ }^{1,2}$ Sascha Husa, ${ }^{3}$ Frank Ohme, ${ }^{4}$ and P. Ajith ${ }^{5,6}$ \\ ${ }^{1}$ Faculty of Physics, University of Vienna, Boltzmanngasse 5, A-1090 Vienna, Austria \\ ${ }^{2}$ School of Physics and Astronomy, Cardiff University, Cardiff, CF24 3AA, United Kingdom \\ ${ }^{3}$ Departament de Física, Universitat de les Illes Balears, Cra. Valldemossa Km. 7.5, Palma de Mallorca, E-07122 Spain \\ ${ }^{4}$ Max-Planck-Institut für Gravitationsphysik, Am Mühlenberg 1, 14475 Potsdam, Germany \\ ${ }^{5}$ LIGO Laboratory, California Institute of Technology, Pasadena, California 91125, USA \\ ${ }^{6}$ Theoretical Astrophysics, California Institute of Technology, Pasadena, California 91125, USA
}

(Received 16 September 2010; published 22 December 2010)

\begin{abstract}
One way to produce complete inspiral-merger-ringdown gravitational waveforms from black-holebinary systems is to connect post-Newtonian (PN) and numerical-relativity (NR) results to create "hybrid" waveforms. Hybrid waveforms are central to the construction of some phenomenological models for gravitational-wave (GW) search templates, and for tests of GW search pipelines. The dominant error source in hybrid waveforms arises from the PN contribution, and can be reduced by increasing the number of NR GW cycles that are included in the hybrid. Hybrid waveforms are considered sufficiently accurate for $\mathrm{GW}$ detection if their mismatch error is below 3\% (i.e., a fitting factor above 0.97). We address the question of the length requirements of NR waveforms such that the final hybrid waveforms meet this requirement, considering nonspinning binaries with $q=M_{2} / M_{1} \in[1,4]$ and equal-mass binaries with $\chi=S_{i} / M_{i}^{2} \in[-0.5,0.5]$. We conclude that, for the cases we study, simulations must contain between three (in the equal-mass nonspinning case) and ten (the $\chi=0.5$ case) orbits before merger, but there is also evidence that these are the regions of parameter space for which the least number of cycles will be needed.
\end{abstract}

DOI: 10.1103/PhysRevD.82.124052

PACS numbers: 04.30.Db, 04.25.D-, 04.25.Nx

\section{INTRODUCTION}

Numerical simulations play a key role in efforts to detect gravitational waves (GWs) from compact-binary coalescences, in particular from black-hole binaries. One of their most direct applications for GW searches has been as input in phenomenological [1-6] and effective-one-body (EOB) [7-15] waveform models, which can in turn be used to construct template banks of theoretical waveforms for use in matched-filter searches in detector data. As part of the NINJA project [16] they are also used to test a battery of search pipelines: the numerical-relativity (NR) waveforms are injected into simulated detector noise and the search pipelines attempt to find them $[17,18]$.

Waveform models are constructed by combining information from post-Newtonian (PN) calculations of the long slow inspiral of the binary [19], and NR simulations of the last orbits and merger. The PN approximation becomes less accurate as the binary approaches merger, and we will argue in this paper that, at the current state of the art, uncertainties in the PN waveforms dominate significantly over errors in the NR waveforms. Therefore more accurate waveform models can be produced by using less PN inspiral cycles, and correspondingly more NR cycles. The question we begin to address in this paper is: how many NR cycles are necessary in order to produce waveform models that are sufficiently accurate for GW detection? By this we mean that the mismatch error in the full waveform (minimized with respect to all search parameters) is less than $3 \%$, as described in Sec. II.
This question is important because NR waveforms from an extremely large number of binary configurations may be necessary to produce waveform models that accurately represent the entire black-hole-binary parameter space. NR simulations are computationally expensive, and the computational cost grows drastically with the length of the simulation. If the number of inspiral cycles in a simulation is to be increased by a factor of 2 , for example, the simulation does not only have to run for twice as long. To accurately capture the phase evolution of the binary over this extended time, higher numerical resolutions are required, and the overall increase in the cost of the simulation may be by factors of 10 , both in memory usage and the time the simulation takes to run.

Current "long" simulations cover $\sim 10 \mathrm{GW}$ cycles before merger [20], and the longest simulation to date covers about 30 cycles [21]. The task of exploring the full blackhole-binary parameter space with long numerical simulations is a large-scale computational challenge that will take several years with current methods. On such a time scale, it is crucial to understand as much as possible about any requirements that may affect the overall cost by an order of magnitude.

The necessary length of NR waveforms will change with improvements in the accuracy of PN methods and increased understanding of EOB methods. As such, we can only make a first step in considering waveform length requirements. Our method is to study hybrid waveforms, which are a simple connection of PN and NR results, and 
allow us to focus on the effects of the uncertainties in PN waveforms without having to take into account any additional artifacts that might arise in a given waveform-model construction procedure. For the purposes of this paper, PN-waveform uncertainties are estimated by the differences between two common PN approximants. We believe that this allows us to make the most conclusive statements that are possible with the current state of the art.

We summarize mismatch calculations in Sec. II, describe our numerical waveforms in Sec. III, and the production of hybrid waveforms in Sec. IV. We use hybrids based on two time-domain PN approximants, the TaylorT1 and TaylorT4 approximants, the accuracy of which we have already studied over a ten-cycle range before merger in [22]. We put our previous PN-NR comparisons into the context of GW searches by computing the mismatch between hybrids with differing numbers of TaylorT1 and NR cycles in Sec. V, and demonstrate that the hybrid errors are indeed dominated by PN effects. We then turn to the question of length requirements for NR waveforms.

We will explain and justify our approach in Sec. VI. Since it has already been shown that pure PN waveforms truncated before merger are sufficient to detect nonspinning binaries with masses up to $12 M_{\odot}$ [23], we consider binaries with total mass $M>10 M_{\odot}$, and our analysis is with respect to the Advanced LIGO detector [24]. Our study covers a significant portion of the parameter space that has already been treated by NR simulations: nonspinning binaries with mass ratios up to $q=M_{2} / M_{1}=4$, and equal-mass binaries with equal, nonprecessing spins up to $\chi= \pm 0.5$. Results for nonspinning binaries are given in Sec. VII, and spinning binaries are treated in Sec. VIII. We will not consider phenomenological or EOB methods, and we will only briefly touch on parameter estimation in Sec. IX. Our first conclusions about waveform length requirements are summarized and discussed in the Conclusion.

\section{MISMATCHES AND GW DETECTION}

The quantity that we will use to assess the detectability of our hybrid waveforms is the mismatch. The standard requirement for current $\mathrm{GW}$ searches is that the members of a template bank be separated such that the no more than $10 \%$ of signals would be lost due to the mismatch between the signal and the template, and this translates into a mismatch between neighboring templates of less than $3 \%$ $[25,26]$. However, one must also take into account the mismatch due to errors in the theoretical waveforms; ultimately we want the sum of the template-spacing mismatch and the waveform-error mismatch to be less than 3\%. We are then free to decide how to divide that mismatch between template spacing and waveform error. For example, the authors of the waveform accuracy study in [27] request that the waveform-error mismatch be below $0.5 \%$. We could alternatively argue that the waveform error can be close to $3 \%$, and the template spacing should be made correspondingly small. This question deserves further attention, but in this work we will consider 3\% as the basic requirement, and also quote results consistent with a maximum mismatch requirement of $1.5 \%$ and $0.5 \%$.

For two GW signals $h_{1}$ and $h_{2}$, we define an inner product in the Fourier domain weighted by the power spectral density of the detector noise, $S_{n}(f)$, as [28]

$$
\left\langle h_{1} \mid h_{2}\right\rangle:=4 \operatorname{Re}\left[\int_{f_{\min }}^{f_{\max }} \frac{\tilde{h}_{1}(f) \tilde{h}_{2}^{*}(f)}{S_{n}(f)} d f\right],
$$

where $\left[f_{\min }, f_{\max }\right]$ is the intersection of the chosen sensitivity range of the detector $\left(\left[20,10^{4}\right] \mathrm{Hz}\right.$ in this study) and the range of validity of the waveform data (in most cases $\left.f M \in\left[1.25 \times 10^{-3}, 0.15\right]\right) . \tilde{h}_{1}(f)$ and $\tilde{h}_{2}(f)$ denote the Fourier transform of $h_{1}$ and $h_{2}$, respectively. Our data in general represent the Weyl scalar $\Psi_{4}(t)$, not the wave strain $h(t)$, but the two are related by $\Psi_{4}=\ddot{h}_{+}-i \ddot{h}_{\times}$, and two time integrations can be performed trivially in the frequency domain.

Given the definition of the inner product $\left\langle h_{1} \mid h_{2}\right\rangle$, we normalize it and maximize over phase and time offsets in the data. This is the faithfulness of the waveform: it is a measure of how "far" a theoretical waveform is from a supposedly true waveform with the same physical parameters. The faithfulness mismatch is the deviation of the faithfulness from unity:

$$
\mathcal{M}=1-\max _{\tau, \Phi} \frac{\left\langle h_{1} \mid h_{2}\right\rangle}{\sqrt{\left\langle h_{1} \mid h_{1}\right\rangle\left\langle h_{2} \mid h_{2}\right\rangle}} .
$$

In a GW search, the goal is essentially to find the template-bank member that has the smallest mismatch with the detector data. We are therefore really interested in the mismatch optimized over all of the members of a theoretical waveform family, i.e., minimized with respect to the binary's intrinsic physical parameters (the mass, mass ratio, and spins), and the extrinsic parameters (the position of the binary in the sky and its orientation). The fully optimized mismatch is the same as $(1-F)$, where $F$ is the waveform's fitting factor. In our study we have access to signals of isolated binary configurations, and the only parameter we can optimize with respect to is the total mass. As such, an optimization of the mismatch with respect to total mass can be no more than an upper bound on the full optimized mismatch. But we will argue in Sec. VII that optimization with respect to the other physical parameters will not qualitatively alter our results. In Secs. IV and V, where we assess the sources of physical error in our hybrids, we will not optimize with respect to total mass. But in Secs. VI, VII, VIII, and IX, where we make calculations relevant to GW detection, we will use the mass optimization, and intend these results to be a reasonable estimate of the mismatch calculated from the fully optimized fitting factor.

Our main focus in this work is GW detection, but we will also refer to a quantity that is relevant to parameter 
estimation. If we have two theoretical waveforms for the same physical system, $h_{1}$ and $h_{2}$, each with some associated uncertainty, then we can define $\delta h=h_{1}-h_{2}$ and then $\|\delta h\|^{2}=\langle\delta h \mid \delta h\rangle$. If $\|\delta h\|<1$, then the two waveforms would be indistinguishable in a detector measurement [27]. The indistinguishability of two waveforms depends on the signal-to-noise ratio (SNR) of the detection: if the SNR is sufficiently low, then any two waveforms will be indistinguishable (although below an SNR of eight they will not be considered detectable anyway), and for two waveforms of arbitrarily low $\|\delta h\|$, we can always find an SNR high enough such that they can in fact be distinguished. For example, in [29] we found that current NR equal-mass nonspinning waveforms from five different codes would be indistinguishable in the Advanced groundbased detectors if the SNR is below 25. In [5] it was shown that $q=2$ hybrid waveforms produced with the BAM and LLAMA [30,31] codes are indistinguishable for an SNR below roughly 20 .

The mismatch error between two waveforms can be easily related to $\|\delta h\|$ [32]. If $\rho=\|h\|$ is the optimal SNR, then $\|\delta h\| / \rho^{2} \approx 2 \mathcal{M}$. This means that if two waveforms meet the detection criteria of $\mathcal{M}<0.03$, then they will be indistinguishable for $\rho<4$, which is too weak a signal to be detected. If we want the waveforms to be indistinguishable at an SNR of $\rho>8$, then the mismatch must be below about $0.8 \%$. We see, then, that the accuracy requirements for two waveforms to be indistinguishable are in general far more stringent than those for detection. We will return to this point in Sec. IX.

All of the results in this work will be with respect to the Advanced LIGO detector [24,33], and in general we will use a low-frequency cutoff of $20 \mathrm{~Hz}$.

\section{NUMERICAL WAVEFORMS}

We consider two families of black-hole binaries. The first is equal-mass binaries in which the spin of each black hole is the same, $S_{1} / M_{1}^{2}=S_{2} / M_{2}^{2}=\chi$, and the spins are parallel or antiparallel to the orbital angular momentum. In these configurations the spins do not precess, making this a simple subfamily of the black-hole-binary parameter space. The spins used were $\chi=\{0, \pm 0.25, \pm 0.50, \pm 0.75$, $\pm 0.85\}$, although we will only provide length requirements up to $\chi= \pm 0.5$ for reasons that will be explained in Sec. VIII. The second family is nonspinning binaries with mass ratios $q=M_{2} / M_{1}=\{1,2,3,4\}$, where we labeled the individual masses such that $M_{2} \geq M_{1}$.

The simulations were produced using the BAM code $[34,35]$. They cover $6-10$ orbits before merger. The phase error of the waveforms is at most 0.15 rad during the inspiral phase (up to $M \omega=0.1$ ), and on the order of $1 \mathrm{rad}$ during merger and ringdown. The amplitude accuracy is within $1 \%$ during the inspiral, and within $5 \%$ during the merger and ringdown. Of more relevance to $\mathrm{GW}$ detection is the mismatch error of the waveforms, which is below
$10^{-4}$ for all cases. Note that this is well within the detection requirement of 0.03 , and indeed these waveforms are well within the accuracy requirements for both detection and parameter estimation with current and planned groundbased detectors [29]. These waveforms were all presented in detail in $[22,36,37]$.

\section{HYBRID INSPIRAL-MERGER-RINGDOWN WAVEFORMS}

Complete inspiral-merger-ringdown waveforms that include arbitrarily large numbers of GW cycles before merger can be constructed by connecting PN and NR results $[1,2,5,38,39]$. We model the inspiral regime using the time-domain approximants TaylorT1 and TaylorT4 [5,37]. For nonspinning binaries the phase evolution is described to 3.5PN order, and the amplitude is given to 3PN order [40]. For spinning binaries, spin effects in the phase evolution are included only up to 2.5PN order [37], and in the case of TaylorT4 we adopt two approaches to truncating the expansion in a consistent way [5,22]; the amplitude includes spin contributions up to $2 \mathrm{PN}$ order [41].

The PN results are expansions in the frequency, $x=$ $(M \Omega)^{2 / 3}$, where $\Omega$ is the orbital frequency of the binary motion. When decomposing the GW signal in spherical harmonics, $\Omega$ is related to the frequency $\omega$ of the $(\ell=2$, $m= \pm 2$ ) modes by $|\omega|=2 \Omega$. As such, the PN waveforms are most accurate for small $x$, i.e., many orbits before merger. As the binary approaches merger, the errors in the PN approximation grow. The convergence properties of the PN expansion are not fully understood, and it is not possible to provide clear error estimates. One way to gain some insight into the PN errors is to compare results from different PN approximants and at different PN orders, and we will return to this idea later.

The only way to definitively quantify the PN errors is to compare with fully general relativistic results. This can be done over the (relatively small) number of GW cycles for which we have both PN and NR waveforms. PN-NR comparisons of phase and amplitude have been performed over $\sim 10$ orbits prior to merger [21,36,42-44]. Comparisons for the cases we consider here are given in [22]. There we found that the TaylorT4 approximant has an accumulated phase error of no more than 0.2 rad for nonspinning cases, but the phase error grows to up to $2 \mathrm{rad}$ for equal-mass cases with large spin, in particular, the $\chi<0$ cases. The behavior of the TaylorT1 phase appears to be most consistent across the parameter space that we consider, with an accumulated phase error of about $1 \mathrm{rad}$ in all cases, although in most cases a version of TaylorT4 is more accurate. The PN amplitude (at the highest-known PN order) has an error as high as $4 \%$ in the $\chi=0.85$ case, and drops to around $2 \%$ in the $\chi=-0.85$ case, while for nonspinning configurations it is around 3\%. (Note, however, that the uncertainty in the NR amplitude for all of these cases is $1 \%$.) The existence of this region of good 
agreement between $\mathrm{PN}$ and NR results is what allows us to combine the two into hybrid waveforms.

We construct time-domain hybrid waveforms by first decomposing the PN and NR waveforms into their amplitude $\left[A_{\mathrm{PN}}(t)\right.$ and $\left.A_{\mathrm{NR}}(t)\right]$ and phase $\left[\phi_{\mathrm{PN}}(t)\right.$ and $\left.\phi_{\mathrm{NR}}(t)\right]$. We only consider clean cycles of the NR waveforms, i.e., we omit the waveform from the early part of the simulation, which contains junk radiation from the initial data and some egregious gauge artifacts. From the phase we can in turn calculate the frequency, $\omega(t)=\partial_{t} \phi(t)$. We then choose a matching frequency, $\omega_{m}$, and determine the time when both $\phi_{\mathrm{PN}}$ and $\phi_{\mathrm{NR}}$ reach that frequency, and connect the two phase functions at that time. We independently determine the time when the PN and NR amplitudes agree, and connect the amplitudes at that time. In this procedure we do not require that the transition between the PN and NR phases and amplitudes is any more than continuous. This procedure is performed on $\Psi_{4}$. In mismatch calculations we first calculate $\tilde{\Psi}_{4}$ by making a fast Fourier transform (FFT) of $\Psi_{4}$, and then produce $\tilde{h}$ by dividing by $-\omega^{2}$.

\section{Errors due to the hybridization process}

There are currently several procedures to produce hybrid waveforms that have been used in the literature, based either on a matching in the time or Fourier domains $[1,2,5,38,39]$. Each procedure will itself introduce artifacts into the waveform, and we may be concerned that these artifacts will be a large source of error. Here we will compare hybrids produced using three different methods, and show that in fact the mismatch error introduced by the hybridization procedure is negligible.

We consider three equal-mass nonspinning hybrids. The first is produced by the method that we have just described. The second is produced using the method described in [1,2], but applied to $\Psi_{4}$ instead of the wave strain $h$, because we want to assess the errors due to the hybridization process, without any contamination by errors that may be introduced by a time-domain integration of $\Psi_{4}$ to $h$. Our specific method is to choose a matching frequency, $\omega_{m}$, and to then locate the time in both the PN and NR waveforms when that frequency is reached. We then combine the two waveforms over a $200 M$-long window, aligning the waveforms such that the quantity

$$
\Delta \Psi=\int_{t_{0}}^{t_{1}}\left(\Psi_{4, \mathrm{NR}}(t)-a e^{i \delta \phi} \Psi_{4, \mathrm{PN}}(t+\delta t)\right)^{2} d t
$$

is minimized, where $t_{0}$ and $t_{1}$ are respectively $100 \mathrm{M}$ before and after the time $t_{m}$ at which each waveform reaches $\omega_{m}, a$ is a scale factor, $\delta t$ and $\delta \phi$ are time and phase offsets, and the waveforms are initially aligned so that $t_{m}$ is the same for both. The hybrid is constructed by making a linear transition between $\Psi_{4, \mathrm{PN}}$ and $\Psi_{4, \mathrm{NR}}$ over the matching window. For both hybrids, we choose a matching frequency of $M \omega_{m}=0.07$.

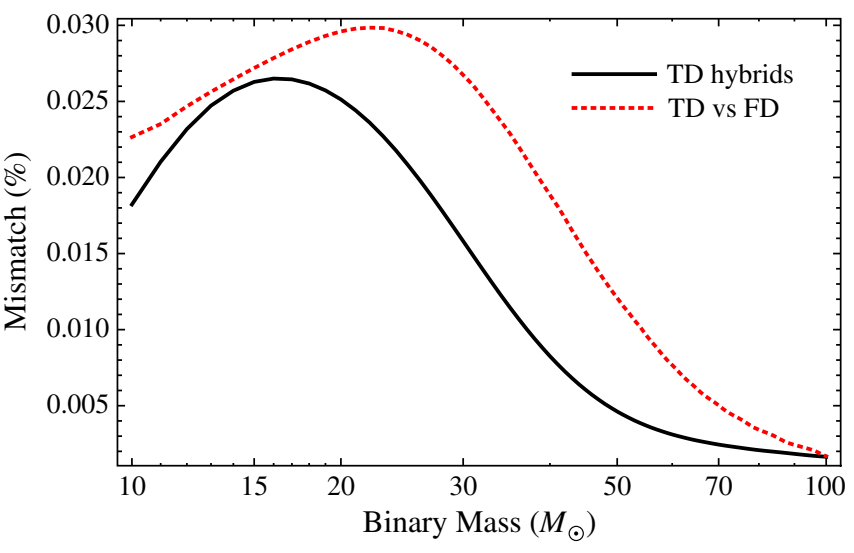

FIG. 1 (color online). Mismatch between equal-mass nonspinning T4 + NR hybrids constructed using two different timedomain methods (solid line), and between hybrids constructed in the time domain (TD) and the frequency domain (FD). The time-domain hybrids are produced with matching frequency $M \omega_{m}=0.07$, and the frequency-domain hybrid is matched at $M \omega=0.079$.

The third hybrid is constructed in the frequency domain, using a variant of the method described in [5]. We produce an FFT of the time-domain TaylorT4 approximant, and an FFT of the numerical $\Psi_{4}$ data. The phase of the frequencydomain PN and NR signals is then matched in the window $M \omega \in[0.0566,0.113]$ and the continuous transition is carried out at the matching frequency $M \omega_{m}=0.079$.

The non-mass-optimized mismatch is shown in Fig. 1. We see that the maximum mismatch is about $0.025 \%$ between the two time-domain hybrids, and $0.03 \%$ between the time-domain and frequency-domain hybrids. Clearly, the error due purely to the hybridization procedure is, like the mismatch error of the numerical waveforms, negligible.

If we consider the indistinguishability criteria, $\|\delta h\|<1$, then these hybrids would be indistinguishable for SNRs of $\rho<40$.

We note, however, that the difference between hybrids constructed with ostensibly the same numerical waveforms and PN approximants may have larger differences than those shown here. For example, if we compare either of the hybrids we have just described, with hybrids constructed using the integrated wave strain, as in $[1,2]$, then the mismatch can be as high as $0.8 \%$. This is due not to the hybridization process, but to artifacts introduced in the time-domain integration of $\Psi_{4}$ to $h$.

\section{COMPARISON BETWEEN PN AND NR DURING THE LATE INSPIRAL}

Direct comparisons between the phase and amplitude of PN approximants and NR waveforms have been made in [21,36,42-46], and we refer the reader to [22] for PN-NR comparisons of the binary configurations studied here. In this section we reframe those comparisons in the context of hybrid waveforms and mismatches. 
For several choices of binary configuration, we construct a fiducial reference hybrid waveform by matching a 3.5PN TaylorT1 waveform to the longest available NR simulation. We then construct a set of "candidate" hybrid waveforms by sliding the matching region over the NR waveform (such that progressively shorter NR segments are employed in the hybrid construction). How well one candidate hybrid waveform can be distinguished from the reference waveform is given by the mismatch between the "reference" and the "candidate."

We study three cases, employing three different NR simulations: an equal-mass, nonspinning simulation performed using the SPEC code [47,48], and two of the BAM simulations presented in [22], equal-mass simulations with spins aligned and antialigned to the orbital angular momentum $(\chi= \pm 0.75)$. Note that in this section, we choose a low-frequency cutoff of $10 \mathrm{~Hz}$, the mismatches are not maximized over the binary mass, and the hybrids are constructed using the procedure described in $[1,2]$.

Figure 2 shows the mismatch between the reference hybrid waveform and the hybrid waveforms constructed using different matching regions in the equal-mass, nonspinning case.

The matching frequency of the reference waveform is $M \omega_{\mathrm{m}, \mathrm{r}}=0.031-0.038$. We will denote the matching frequency of the candidate hybrid by $\omega_{\mathrm{m}}$. When $\omega_{\mathrm{m}, \mathrm{r}}=\omega_{\mathrm{m}}$, then the two waveforms are identical, and the mismatch is zero. As the matching frequency of the candidate waveform $\omega_{\mathrm{m}}$ is increased, the mismatches grow. This gives us a picture of the mismatch due to the disagreement of TaylorT1 and full numerical results over the frequency range $M \omega \in\left[\omega_{\mathrm{m}, \mathrm{r}}, \omega_{\mathrm{m}}\right]$. For the maximum matching frequency we choose, $M \omega_{\mathrm{m}}=0.075$, the maximum mismatch is $3.5 \%$.

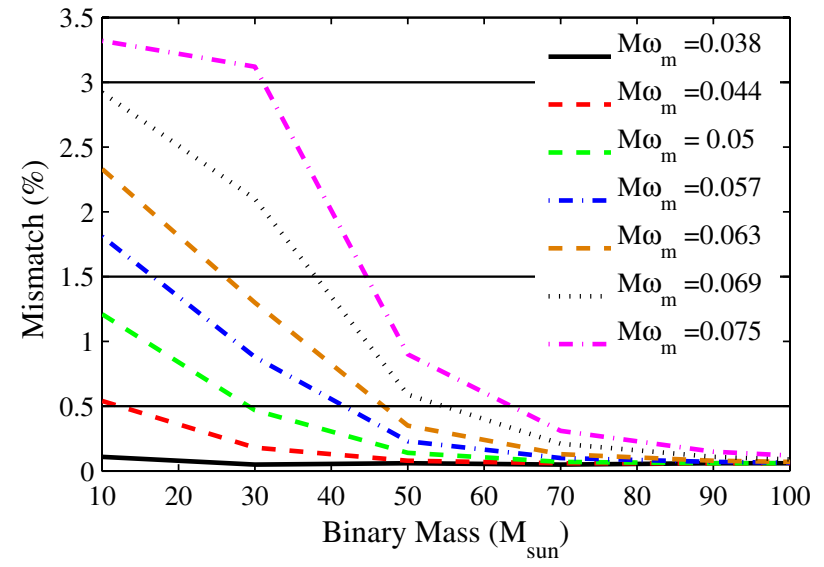

FIG. 2 (color online). Mismatch between the reference hybrid waveform and the hybrid waveforms constructed using different matching frequencies. The hybrid waveforms are constructed by matching TaylorT1 PN waveforms with the Caltech-Cornell equal-mass, nonspinning NR simulation. The PN-NR matching frequency $\omega_{\mathrm{m}}$ of the candidate hybrids is shown in legends.

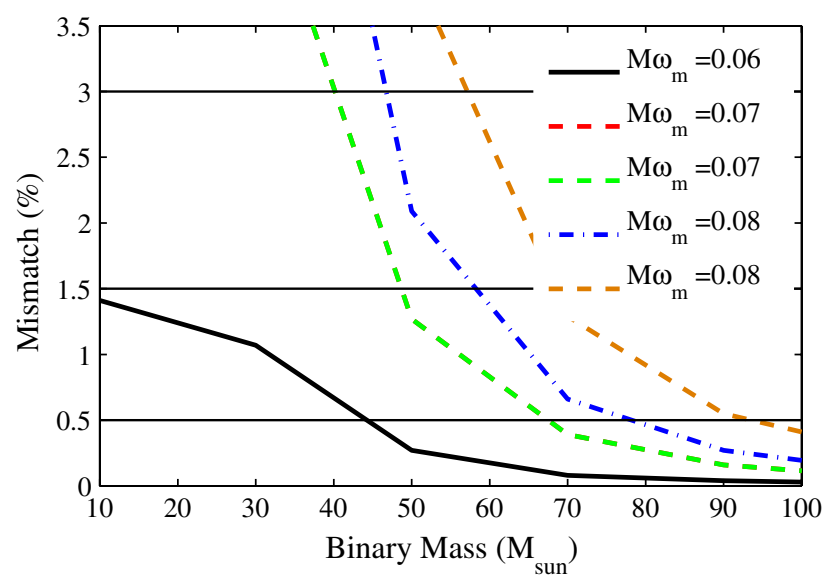

FIG. 3 (color online). Same as in Fig. 2 except that the hybrid waveforms are constructed by matching TaylorT1 waveforms with the BAM simulation of equal-mass binary with spins $\chi=0.75$.

Figures 3 and 4 show the mismatches between the reference and candidate hybrid waveforms for the case of equal-mass binaries with aligned and antialigned spins $\chi= \pm 0.75$, respectively. The $\chi=-0.75$ reference waveform was constructed with a matching frequency of $M \omega_{\mathrm{m}, \mathrm{r}} \simeq 0.038-0.044$, and the $\chi=0.75$ waveform is matched at $M \omega_{\mathrm{m}, \mathrm{r}} \simeq 0.057-0.063$.

We see from these results that the mismatch rises above $3 \%$ at lower matching frequencies than in the nonspinning example. This is a reflection of the fact that the TaylorT1 approximant performs worse in the spinning cases.

These first results give us an indication of the mismatch error associated with the disagreement between the TaylorT1 approximant and NR results over the frequency range for which NR results exist. They show that the accumulation of mismatch error over that frequency range due to PN errors is orders of magnitude larger than the mismatch error due to errors in the numerical waveforms

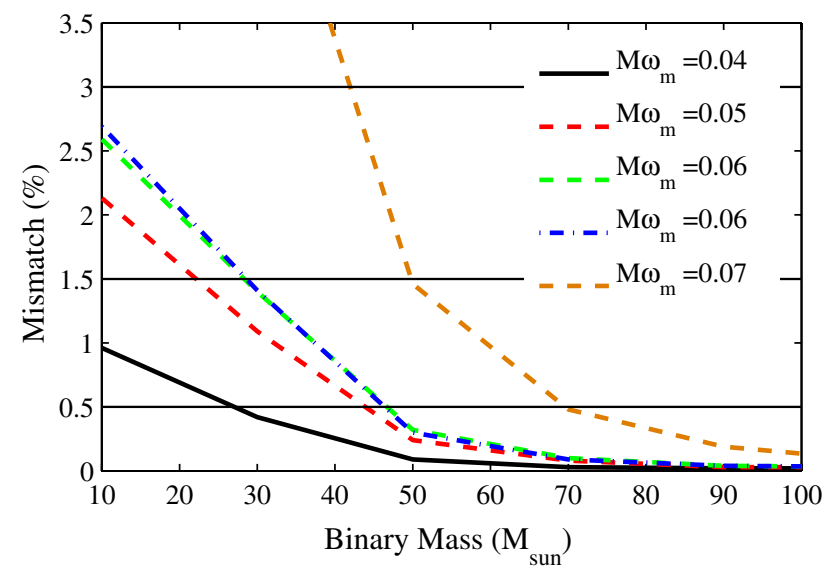

FIG. 4 (color online). Same as in Fig. 2 except that the hybrid waveforms are constructed by matching TaylorT1 waveforms with the BAM simulation of equal-mass binary with spins $\chi=-0.75$. 
or to the hybridization process. These mismatches could drop significantly if we were to minimize the mismatch with respect to binary mass, but that would not alter the qualitative observation that $\mathrm{PN}$ errors dominate the error in our hybrid waveforms.

\section{WAVEFORM LENGTH REQUIREMENTS: GENERAL PROCEDURE}

In producing hybrid waveforms, we have seen that the dominant error source is the error in the PN approximation. We expect the PN waveforms to be increasingly accurate as we move to lower frequencies (i.e., earlier in the inspiral), and so the lower the frequency $\omega_{\mathrm{m}}$ at which we can match (i.e., the more NR cycles we can use) the better. Our goal now is to determine the highest acceptable matching frequency for a variety of accuracy criteria for the full waveforms.

A previous study showed that pure PN approximants of nonspinning binaries (i.e., without any stitching to merger and ringdown signals) are adequate for detection purposes up to about $12 M_{\odot}$ [23]. Therefore our PN + NR hybrids have to be accurate only for total masses $M \gtrsim 10 M_{\odot}$. Above these masses the mismatch between PN inspiral waveforms and full inspiral-merger-ringdown waveforms deteriorates quickly $[23,38,49]$.

The main difficulty in assessing the physical accuracy of hybrids is that, since we cannot rigorously quantify error bars in the PN waveforms, any error estimates we make on our complete waveforms can be no better than an educated guess. The best we can do is to make clear what assumptions have gone into our guess, and to what extent this guess can be considered conservative or optimistic. We will now describe the procedure we have used, and our justifications for it.

Our approach is the following. We start by defining a model waveform that we will regard for the purposes of this study as the "true" physical waveform.

For nonspinning binaries we know that during the frequency range in which PN and NR results overlap, the TaylorT4 approximant captures the phase evolution with high accuracy. We now assume that the TaylorT4 phase continues to be physically correct to much lower frequencies, and we construct a TaylorT4 + NR hybrid and treat that as the true GW waveform from our system. In other words, we treat this hybrid as if it were a numerical waveform starting at $M \omega=0.006$, which corresponds to $20 \mathrm{~Hz}$ for a $10 M_{\odot}$ binary. Such a waveform covers $\sim 650$ cycles before merger. We have no expectation of ever producing such a waveform in a numerical simulation, but for the purposes of this exercise we will treat our hybrid as if it were the result of just such a simulation, and refer to it as $\mathrm{NR}_{\mathrm{L}}$.

Our goal is to assess the accuracy of PN + NR hybrids constructed using a reasonable choice of PN approximant. Based on its phase accuracy near merger, we choose the
TaylorT1 approximant. We construct hybrids of TaylorT1 with $\mathrm{NR}_{\mathrm{L}}$, and due to the great length of $\mathrm{NR}_{\mathrm{L}}$ we have the freedom of matching at arbitrarily low frequencies.

We then calculate the mismatch between $\mathrm{T} 1+\mathrm{NR}_{\mathrm{L}}$ and the true $\mathrm{NR}_{\mathrm{L}}$ waveform, and use this to estimate the ability of $\mathrm{T} 1+\mathrm{NR}_{\mathrm{L}}$ to detect the true signal. This process is repeated for a range of matching frequencies, which allows us to determine the highest matching frequency at which the $\mathrm{T} 1+\mathrm{NR}_{\mathrm{L}}$ hybrid is sufficiently accurate for detection of binaries above $10 M_{\odot}$.

The one key assumption in this procedure is that the TaylorT4 phase can be trusted to much lower frequencies than those where it has currently been compared with full numerical results. The first and most detailed study of TaylorT4 was presented in [21], which included frequencies down to $M \omega \approx 0.035$, so we know that TaylorT4 (at least in the nonspinning case) is accurate down to that frequency. But below that frequency we have no information about its accuracy with respect to waveforms from full general relativity. We expect that the difference between the TaylorT4 and TaylorT1 phases provides a reasonable estimate of the phase error in a typical approximant, but there is no way to prove this. Ultimately one is reduced to a statement of faith in the accuracy of PN methods.

Let us illustrate this point with two extreme views.

Instead of comparing TaylorT1 and TaylorT4 hybrids, we could compare hybrids produced using different effective-one-body (EOB) calculations. It has been claimed that EOB methods, particularly after calibration to numerical results, provide an extremely accurate model of the full GW signal [7-15,50-54]. As with standard PN approximants, EOB results can only be tested in the regime where NR results exist. However, all PN and EOB results make use of expressions for the GW flux and energy loss of the binary, and it has been shown in [55] that EOB estimates of these quantities agree with NR results far better than any standard PN estimates. We might then expect, on this evidence alone, that EOB results are far more accurate than any standard PN approximant, and the appropriate comparison would be between hybrids produced using variants of the EOB method.

This view suggests that the comparison we are proposing - between T1 and T4 hybrids - is overly pessimistic of the potential physical fidelity of hybrid waveforms.

An alternative view is that EOB waveforms (whether the final waveform phase, or the flux and energy-derivative ingredients) have nonetheless only been compared with full general relativity (GR) results close to merger, and their accuracy at lower frequencies is unknown. One could argue that we should be much more conservative, and instead of comparing 3.5PN TaylorT1 and TaylorT4 hybrids, we should compare hybrids constructed using different PN orders. Only by comparing, for example, 2.5PN and 3.5PN results, can we hope to estimate the error between 3.5PN results and those from full GR. As we will see in 
Sec. VII, this provides an extremely pessimistic view of the accuracy of 3.5PN waveforms.

In the end, then, we consider our proposed T1-T4 comparison to be a reasonable compromise between these two extreme views. But we hope this long preamble serves as a caveat that all such comparisons are by no means fully conclusive.

\section{NONSPINNING BINARIES}

We will now investigate the length requirements for simulations of nonspinning binaries. We start with the equal-mass nonspinning case.

We first construct a hybrid between 3.5PN TaylorT4 and our numerical waveform. We make the transition between PN and NR at a matching frequency $M \omega_{m}=0.09$. Figure 5 shows the mismatch between this waveform and a hybrid produced with a different matching frequency, and suggests that for our purposes the resulting hybrid does not depend strongly on the matching frequency $\omega_{m}$, since we are not concerned by mismatches that are below $0.5 \%$. Note that these results are a further reflection of the good agreement of TaylorT4 with the numerical phase at these frequencies; we see from Fig. 2 that the same would not be true for T1 + NR hybrids.

We treat this waveform as if it were an extremely long numerical waveform, and denote it $\mathrm{NR}_{\mathrm{L}}$. This is our target GW signal. It is around $4 \times 10^{5} \mathrm{M}$ in duration, making it 2 orders of magnitude longer than the longest current equal-mass nonspinning waveform [21]. TaylorT1 $+\mathrm{NR}_{\mathrm{L}}$ hybrids are then constructed with a range of matching frequencies, and compared with $\mathrm{NR}_{\mathrm{L}}$, as described in the previous section.

Figure 6 shows the mismatch between $\mathrm{NR}_{\mathrm{L}}$ and $\mathrm{T} 1+$ $\mathrm{NR}_{\mathrm{L}}$ hybrids for $M \omega_{m}=0.045,0.05,0.06,0.07,0.09$. Above the highest matching frequency of $M \omega_{m}=0.09$

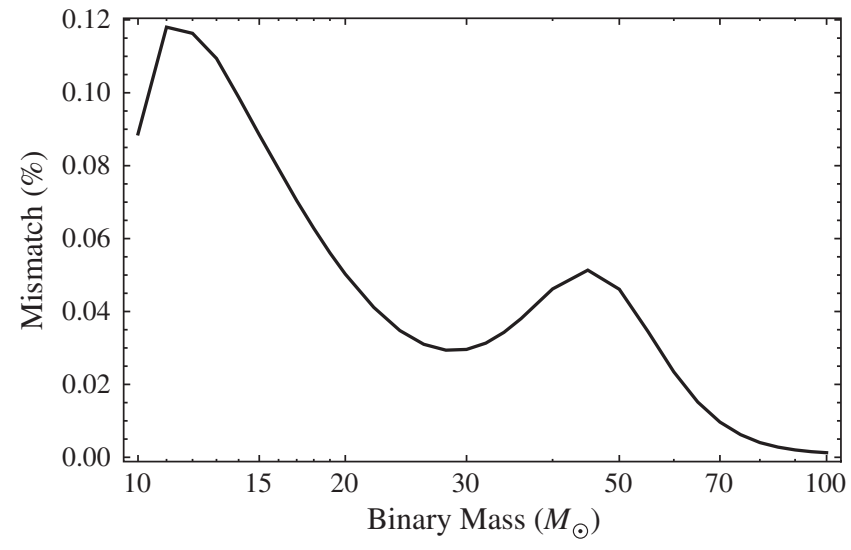

FIG. 5. Mismatch between T4 + NR hybrids constructed with matching frequencies $M \omega_{m}=0.06$ and $M \omega_{m}=0.09$. We see that the mismatch between T4 hybrids constructed at different matching frequencies is below $0.12 \%$ over the entire mass range that we consider.

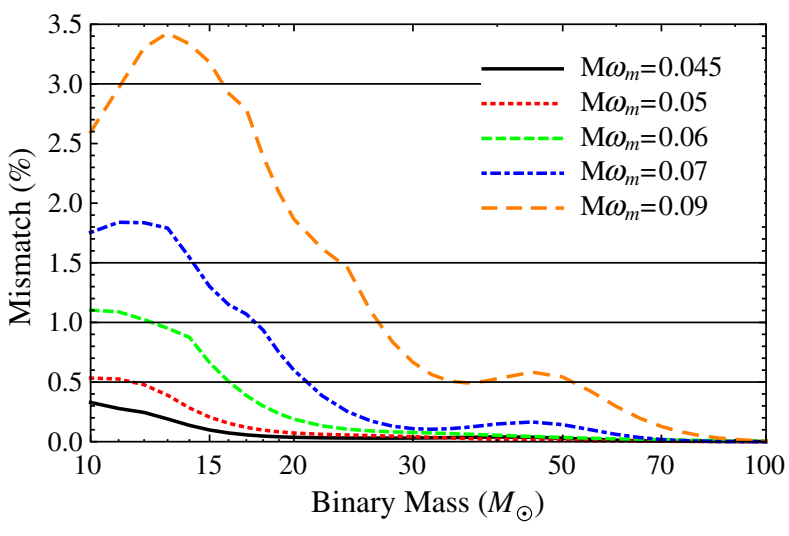

FIG. 6 (color online). Mismatches between $\mathrm{T} 1+\mathrm{NR}_{\mathrm{L}}$ and $\mathrm{NR}_{\mathrm{L}}$ hybrids, for matching frequencies $M \omega_{m}=\{0.045,0.05$, $0.06,0.07,0.09\}$.

both hybrids are identical for all the matching frequencies we have chosen. This means that for masses above about $150 M_{\odot}$, the two waveforms will be almost identical within the sensitivity range of the detector, and their mismatch should approach zero. For this reason Fig. 6 considers masses only up to $100 M_{\odot}$, and indeed we see that the mismatch has essentially dropped to zero by $100 M_{\odot}$.

At lower masses, the difference between the two PN approximants dominates, and the mismatch rapidly grows. At yet lower masses, the PN approximants are being sampled at lower frequencies, where their agreement is better, and we expect the mismatch to fall. This behavior can be seen for the matching frequency $M \omega_{m}=0.09$, where the peak mismatch is at about $13 M_{\odot}$. For lower matching frequencies, the peak mismatch is pushed to lower masses, and for $M \omega_{m} \leq 0.05$ we do not see it in the figure. We expect that the location of the mismatch maximum is related to the mass at which $M \omega_{m}$ is at the detector's most sensitive frequencies. The detector's peak sensitivity is at around $200 \mathrm{~Hz}$, and for example $M \omega=$ 0.09 corresponds to that frequency for $M=15.4 M_{\odot}$, which is consistent with Fig. 6.

As described in Sec. VI, the mismatch is optimized with respect to the total mass of the binary. We cannot optimize over the mass ratio, because we do not have access to numerical waveforms with $q$ close to unity. However, we can make a crude estimate of the magnitude of the effect of mass-ratio optimization. If we consider a $10 M_{\odot}$ binary, then most of the hybrid in the detector's sensitivity band is from the PN contribution. We vary the mass ratio of a TaylorT1 waveform until its phase agreement with TaylorT4 is optimized, and then construct a TaylorT1 + $\mathrm{NR}_{\mathrm{L}}$ hybrid using that mass ratio, and repeat the above analysis with $M \omega_{m}=0.06$. The optimal mass ratio is $q=$ 0.99985 , and the change in the mismatch is indistinguishable on the scale of Fig. 6. This suggests that the mass optimization by itself is sufficient to give an indication of the full mismatch error of our hybrids. 
If we are willing to consider waveforms acceptable so long as the waveform-error mismatch is below $3 \%$, then we see from Fig. 6 that the $M \omega_{m}=0.09 \mathrm{~T} 1$ hybrids are almost good enough, and hybrids produced with any lower matching frequency are acceptable. With this criterion, we need waveforms that contain only five $\mathrm{GW}$ cycles before merger, or about two orbits. (Note that the number of GW cycles before the peak amplitude is more than simply twice the number of orbits, because the approximate quadrupole relation $\omega=2 \Omega$ no longer holds.)

Being more realistic, and demanding that the mismatch is below $1.5 \%$ (so that the remainder of our allowed $3 \%$ mismatch is taken up by the template spacing), a matching frequency of $M \omega_{m}=0.06$ is more than acceptable. This means that we need about $11 \mathrm{GW}$ cycles before merger, or about five orbits. If we are especially stringent and require that the error mismatch be below $0.5 \%$, then we must match below $M \omega_{m}=0.05$, and this requires 15 cycles (seven orbits).

For the equal-mass nonspinning case, then, we see that the NR-waveform length requirements for GW detection are not very high: five orbits are sufficient, and seven orbits are plenty.

At this point we may ask what happens if we decide to be much more conservative in estimating the PN error. We can do this by repeating the exercise above, but instead using TaylorT1 waveforms at only $2.5 \mathrm{PN}$ order. The results are shown in Fig. 7. We see that the variation in the mismatch is dramatic. Even if we choose a matching frequency of $M \omega_{m}=0.04$ (which corresponds to $23 \mathrm{GW}$ cycles before merger), the waveforms are not accurate enough for searches, irrespective of template-bank spacing, for masses below $15 M_{\odot}$. Only if we match at $M \omega=0.03$ (40 cycles before merger) are the waveforms usable, and to get close to the more rigorous mismatch requirement of $1.5 \%$ for all masses, we need $M \omega=0.02$, which corresponds to $80 \mathrm{GW}$ cycles before merger. If one is to adopt the view that this is a realistic estimate of the uncertainty in our

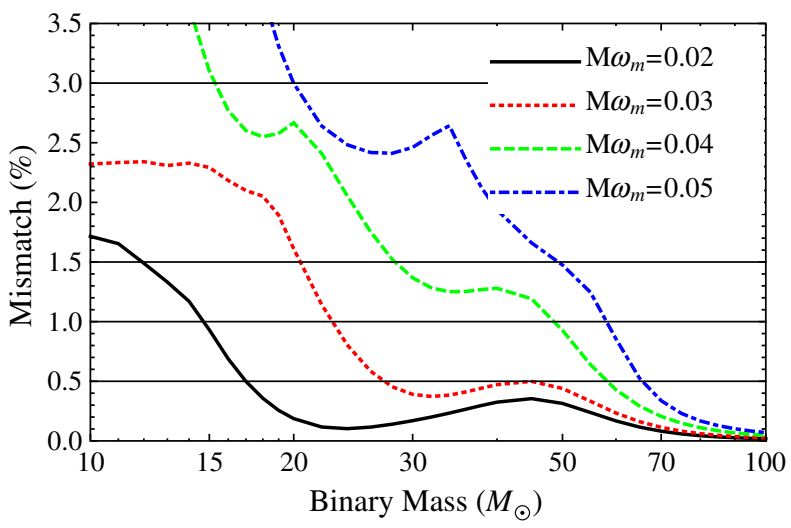

FIG. 7 (color online). Mismatches between $\mathrm{T} 1+\mathrm{NR}_{\mathrm{L}}$ and $\mathrm{NR}_{\mathrm{L}}$ hybrids, where the TaylorT1 approximant is evaluated at only $2.5 \mathrm{PN}$ order.
3.5PN approximants, then we must conclude that hybrids constructed from even the longest numerical waveforms currently published are only useful for searches down to 15-20 solar masses. It is the opinion of the authors, however, that this is a gross exaggeration of the error in $3.5 \mathrm{PN}$ approximants.

We now consider higher mass ratios. The results in [22] suggest that TaylorT4 is also a good model for the true waveform for the mass ratios that we consider, $q=$ $\{1,2,3,4\}$. We find that the maximum acceptable matching frequency drops as the mass ratio increases, so that we need longer numerical waveforms. Figure 8 shows the results for $q=4$, which is the most extreme case. The figure shows results with matching frequencies $M \omega_{m}=$ $\{0.04,0.045,0.05,0.06,0.07\}$. Clearly matching frequencies of $M \omega=0.07$ will not be sufficient even if we allow mismatches up to $3 \%$, but $M \omega_{m}=0.06$ (15 cycles, or about 7 orbits before merger) is borderline. A matching frequency of $M \omega_{m}=0.05$ (21 cycles, 10 orbits) ensures mismatches no higher than $1.5 \%$, and mismatches below $0.5 \%$ require a matching frequency of $M \omega=0.04$ (33 cycles, or about 15 orbits).

We see, then, that for higher mass ratios it is not sufficient to produce waveforms of the same modest lengths as in the equal-mass case. This is unfortunate, because higher-massratio simulations are far more computationally expensive. This result also highlights a drawback of direct comparisons between NR and PN results over a small number of cycles before merger: in such comparisons, the performance of TaylorT1 and TaylorT4 is roughly the same for $q=1$ and $q=4$, and we might therefore conclude that the length requirements for NR waveforms would be the same if we want to produce sufficiently accurate TaylorT1 + NR hybrids in either case. But these results show otherwise; we need more cycles for higher mass ratios.

The $q=4$ numerical waveform used for this study covers $17 \mathrm{GW}$ cycles before merger, and so would in principle be usable for searches down to $10 M_{\odot}$, although

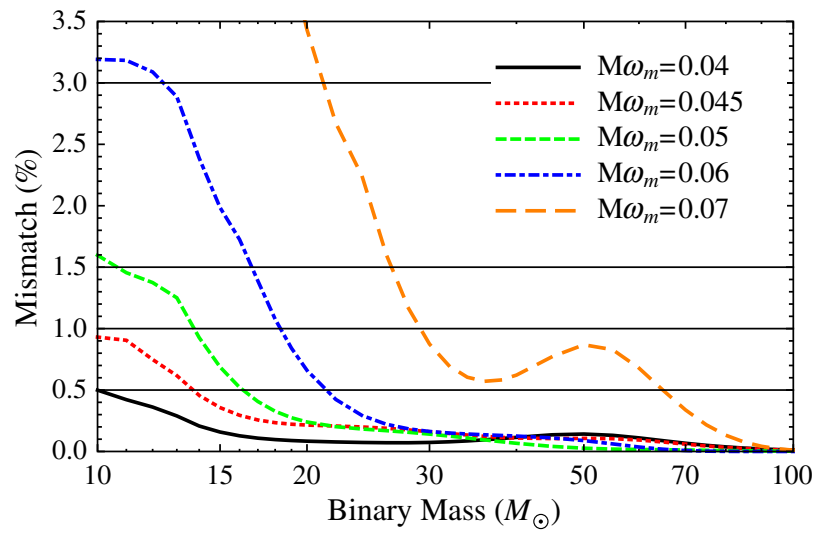

FIG. 8 (color online). Mismatches between $\mathrm{T} 1+\mathrm{NR}_{\mathrm{L}}$ and $\mathrm{NR}_{\mathrm{L}}$ hybrids for $q=4$, with matching frequencies $M \omega_{m}=$ $\{0.04,0.045,0.05,0.06,0.07\}$. 
a slightly longer waveform (for example including ten clean inspiral cycles) would be preferable. It is clear, however, that (1) producing acceptable $q=4$ waveforms is certainly feasible with current codes, but (2) it is nontrivial to estimate the length requirements for yet higher mass ratios.

As a final point, note that for matching frequencies above $M \omega_{m}=0.06$, the mismatch is above $3 \%$ for binaries at $10 M_{\odot}$. This appears to be in contradiction with the results in [23], where it was found that PN inspiral waveforms have a mismatch error below 3\% for masses below $12 M_{\odot}$. This illustrates that mismatches between hybrid waveforms may be higher than those between pure PN waveforms even at relatively low masses, because any optimization in the alignment of the PN part of two hybrids will introduce a dephasing in the NR part, and increase the mismatch. As we have already discussed, this effect decreases for lower masses, but nonetheless means that two $10 M_{\odot}$ hybrids will usually have a higher mismatch that two $10 M_{\odot} \mathrm{PN}$ waveforms. We will discuss this point in further detail in future work.

\section{EQUAL-MASS SPINNING BINARIES}

We now tackle the cases with spin. The TaylorT4 approximant no longer accurately tracks the true phase in the last cycles before merger, and it would be difficult to justify its use in constructing an $\mathrm{NR}_{\mathrm{L}}$ hybrid as in the previous section. (See Figs. 7 and 8 in [22].)

Without a promising approximant to use to produce a stand-in for a true waveform, our next-best option is to construct one. The nonspinning terms of TaylorT4 are known up to 3.5PN order, but the spinning terms are known only up to $2.5 \mathrm{PN}$. Perhaps if we knew the $3 \mathrm{PN}$ and $3.5 \mathrm{PN}$ contributions, the spinning TaylorT4 would be just as good as the nonspinning version? Whether this is true or not, we can certainly introduce 3PN and 3.5PN terms that improve its agreement with numerical results. Our procedure is to adjust the coefficients of such terms so as to minimize the square-integral phase difference between the PN and NR waveforms, as defined by [22]

$$
\overline{\Delta \phi}\left(t_{N}\right)=\frac{1}{\sqrt{-t_{N}}}\left[\int_{t_{N}}^{t_{0}}\left(\phi_{\mathrm{NR}}(t)-\phi_{\mathrm{PN}}(t)\right)^{2} d t\right]^{1 / 2},
$$

where $N$ is the number of cycles included in the comparison, and we choose $N=10$, and the final time $t_{0}$ is the time that corresponds to the GW frequency at which we end our comparison, which in these cases is $M \omega=0.1$. We find in practice that the phase evolution is almost identical using an approximant with either an optimized 3PN coefficient only, or both 3PN and 3.5PN coefficients. This suggests that our fitting procedure over $10 \mathrm{GW}$ cycles is not very sensitive to higher-order coefficients.

However, the minimization process is sensitive to the initial guess for the coefficients, and it is possible that other choices are possible with similar results. The particular
TABLE I. Empirically calculated 3PN and 3.5PN coefficients that produce a TaylorT4 approximant that agrees well with the NR phase over the last ten cycles up to $M \omega=0.1$.

\begin{tabular}{lcc}
\hline \hline$\chi$ & $3 \mathrm{PN}$ & $3.5 \mathrm{PN}$ \\
\hline-0.85 & 989.4 & 16.26 \\
-0.75 & 775.3 & 21.11 \\
-0.50 & 259.2 & 23.99 \\
-0.25 & 39.73 & -1.22 \\
0 & 0.868 & -0.111 \\
0.25 & -128.9 & 0.320 \\
0.50 & -204.6 & 1.070 \\
0.75 & -298.5 & 38.97 \\
0.85 & -268.6 & -1.009 \\
\hline \hline
\end{tabular}

coefficients that we use are given in Table I. We note that the 3PN coefficients depend roughly monotonically on the spin of the black holes, but we will not attempt to infer any significance on the particular values that we obtain. It is important to bear in mind also that these coefficients are chosen to achieve the best agreement over only ten $G W$ cycles, and those cycles are near merger, where the PN approximation is close to breaking down. The "correct" 3PN and 3.5PN coefficients, when they are derived analytically, will be such that they will be expected to lead to a GW phase that is physically correct at low, not high, frequencies. The coefficients that we are using may have the reverse properties, and lead to a poor estimate of the phase at low frequencies-and in fact our ad hoc coefficients may adversely distort the phase function during the early inspiral. If anything, this will lead us to conclude that our waveforms should be much longer than they really need to be, and so our results will still provide an upper bound on the necessary waveform length. And this is our goal.

This point is illustrated in Fig. 9, which shows the accumulated phase disagreement between the optimized 3.5PN TaylorT4 approximant, and our three standard

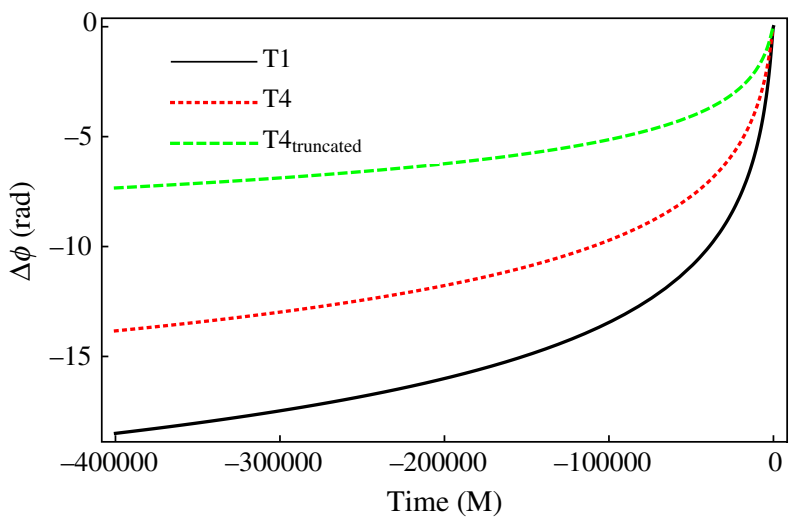

FIG. 9 (color online). Phase disagreement between the optimized 3.5PN TaylorT4 approximant, and three other approximants: TaylorT1, T4, and $\mathrm{T} 4_{\text {truncated }}$, for the $\chi=0.5$ case. The time $t=0$ corresponds to the frequency $M \omega=0.06$, where the phases are lined up. 
approximants, TaylorT1, TaylorT4, and TaylorT4 $4_{\text {truncated }}$ for the $\chi=0.5$ case. The results in [22] show that T4 agrees best with the NR data over the ten cycles of comparison, $\mathrm{T} 1$ is the next best, and $\mathrm{T} 44_{\text {truncated }}$ performs worst. But if we look now at Fig. 9, which shows the phase disagreement during all of the early inspiral up to $M \omega=$ 0.06 , we get a different impression. Now the $\mathrm{T} 4_{\text {truncated }}$ approximant performs best, while T4 is next best, and T1 is the worst. This confirms our suspicion that the relative performance of different approximants during the last orbits of the binary may be quite different to their performance during the earlier inspiral - but it also tells us that if we compare the hybrids produced by the optimized 3.5PN T4 approximant with TaylorT1 hybrids, then this will give us the most conservative estimate of the length requirements of our waveforms. This is because it is the phase disagreement that dominates the mismatch, and Fig. 9 suggests that the $\mathrm{T} 1-\mathrm{T} 4_{3.5 \mathrm{PN}}$ mismatches will be the worst.

We will focus on two cases, $\chi=-0.5$ and $\chi=0.5$. The mismatch plots for these cases are shown in Fig. 10. The most notable aspect of these two plots is the dramatic difference in the mismatches as a function of matching frequency between the two cases. For the anti-hangup case $\chi=-0.5$, a matching frequency of about $M \omega_{m}=0.055$ appears to be sufficient to achieve mismatches below $1.5 \%$.
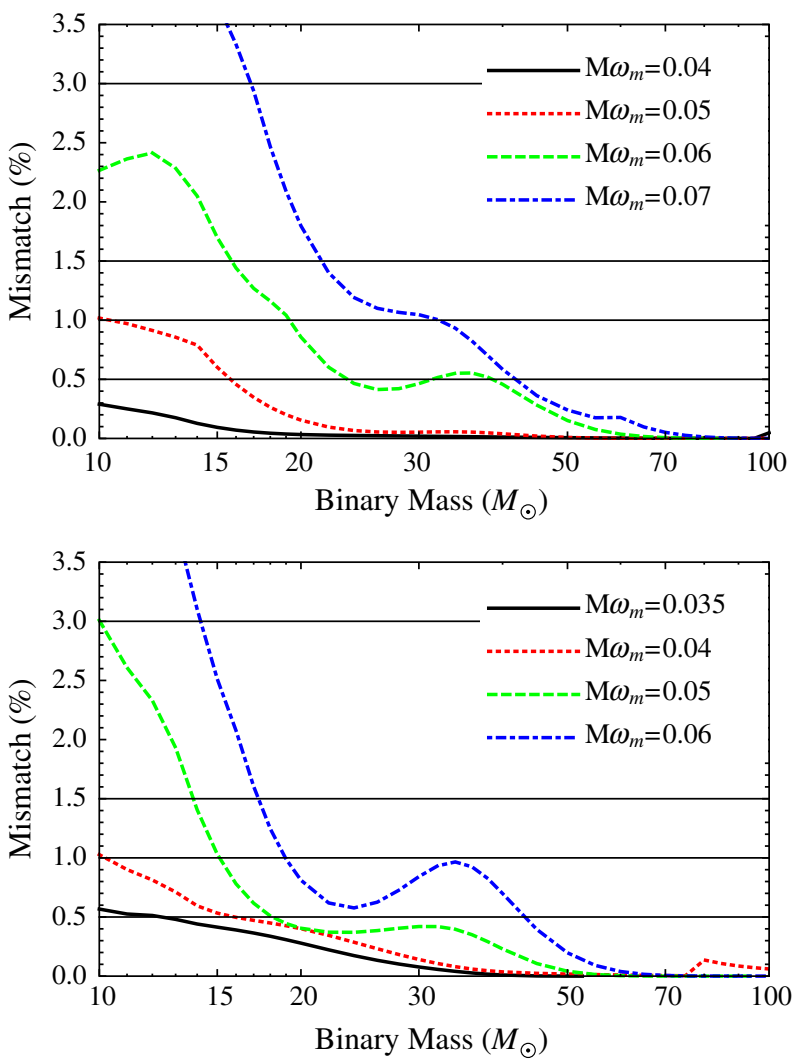

FIG. 10 (color online). Mismatches between T1 and T4 hybrids for the cases $\chi=-0.5$ (upper panel) and $\chi=0.5$ (lower panel).
This corresponds to about ten cycles before merger, and is comparable to what we saw in the nonspinning case. For the hangup case $\chi=0.5$, on the other hand, a matching frequency of between 0.04 and 0.05 is necessary. A matching frequency of $M \omega_{m}=0.04$ corresponds to 28 cycles before merger, or 13 orbits. Some of this difference is due to the effect of spin on the rate of inspiral: from a given frequency, there will be more cycles before merger in the hangup case than in the anti-hangup case. Since the hangup case requires matching at a lower frequency, this adds yet more cycles to our estimate - and so we end up with almost a factor of 3 difference in the number of NR cycles that are required.

To test the robustness of our results, we have also calculated the best-fit modifications to the 3PN and 3.5PN by optimizing over a smaller number of NR cycles. There is a large variation in the parameters as the number of included NR cycles is changed, but we have found that the change in the overall mismatch results is at a level that would not be distinguishable in the figures.

Table II summarizes the number of cycles required before merger for the different cases, for different levels of mismatch requirement. Spins up to only $|\chi|=0.5$ are used, because in higher-spin cases the deviation of the known T4 approximant from the NR phase is so large that the required $3 \mathrm{PN}$ and 3.5PN coefficients appear to distort the overall phase evolution too severely. We can infer from this table, however, that for $\chi<-0.5$, simulations that include $20 \mathrm{GW}$ cycles before merger (ten orbits) should allow the construction of $\mathrm{T} 1$ hybrids that are within the 3\% mismatch accuracy requirement, but for high $\chi>0.5$ many more cycles may be required.

The table also indicates, assuming that we will allow a maximum error mismatch up to $3 \%$, the minimum mass that can be searched for using hybrids constructed from the numerical waveforms presented here. These numbers are provided as a snapshot of what can be done with the waveforms that exist as of the writing of this paper. In most cases

TABLE II. Summary of mismatch calculations. The second, third, and fourth columns give the minimum number of numerical GW cycles before merger that ensure mismatches below $3 \%$, $1.5 \%$, and $0.5 \%$ for all masses above $10 M_{\odot}$. The last column indicates the lowest mass for which the numerical waveforms studied here could be used for searches, assuming that the mismatch error can be as high as $3 \%$.

\begin{tabular}{lrccc}
\hline \hline Configuration & $\mathcal{M}<3 \%$ & $\mathcal{M}<1.5 \%$ & $\mathcal{M}<0.5 \%$ & $M_{\min } / M_{\odot}$ \\
\hline$\chi=-0.5$ & 8.0 & 10.0 & 19.0 & 10 \\
$\chi=-0.25$ & 10.0 & 15.0 & 20.0 & 10 \\
$\chi=0$ & 7.0 & 9.5 & 15.0 & 10 \\
$\chi=0.25$ & 13.0 & 18.0 & 26.0 & 10 \\
$\chi=0.5$ & 20.0 & 26.0 & 36.0 & 15 \\
$q=2$ & 8.5 & 11.5 & 25.0 & 10 \\
$q=3$ & 11.0 & 15.5 & 25.0 & 10 \\
$q=4$ & 15.0 & 21.0 & 33.0 & 10 \\
\hline \hline
\end{tabular}


we can produce hybrids that are acceptable for searches down to $10 M_{\odot}$, but for some spinning cases we can use our hybrids to search down to only $15 M_{\odot}$. We hope that the results in this paper ultimately indicate upper bounds on the required length of numerical waveforms for GW detections. It should be clear from Table II that an improvement in PN approximants (due, for example, to the calculation of higher-order spin terms) would have a significant effect on the NR-waveform length requirements. If the accuracy of spinning approximants were improved to the level of their equal-mass nonspinning counterparts, then numerical waveforms covering only 5-7 orbits before merger would be sufficient for GW searches.

On the other hand, we also see that the length requirements vary significantly between binary configurations, and that more NR cycles are required as we approach the extremes of our parameter choices. We cannot determine from these results what the length requirements would be for $q>4$ nonspinning waveforms, or even for highly spinning waveforms with $q>1$. In this sense, the "worst" cases remain to be studied.

\section{PARAMETER ESTIMATION}

Our focus so far has been on GW detection, and a full parameter-estimation study is beyond the scope of this paper; indeed such a study would require a complete waveform family, and would be more appropriately performed using phenomenological or EOB models. However, we can make some observations about parameter estimation.

As discussed in Sec. II, if for our T1 and T4 hybrids we have $\|\delta h\|<1$, then the two waveforms are indistinguishable. This means that the accuracy of the estimation of the intrinsic parameters of the binary is determined by the SNR of the signal, and not by any error in the waveforms. In other words, if the waveforms are indistinguishable at the SNR of a given measurement, then the maximum parameter information can be extracted from that measurement, and is not limited by the accuracy of the waveforms.

It is reasonable to expect SNRs as high as 30 in Advanced detectors, and for waveforms to be indistinguishable at that SNR, the mismatch error must be below $0.05 \%$ (see again the discussion in Sec. II). It is clear even in the best case (equal-mass nonspinning) that our hybrids meet this criteria only for binary masses higher than $20 M_{\odot}$. To achieve such a low mismatch down to $10 M_{\odot}$ would require numerical waveforms far longer than any that have yet been produced. We have also seen that the mismatch in the hybrids due to artifacts from the hybridization process alone are at $0.03 \%$, and so producing hybrids that are indistinguishable for parameter estimation up to an SNR of 30 is a challenge irrespective of the problems of PN errors and waveform length.

However, even though the hybrids we have presented may not be indistinguishable at the potential SNRs of

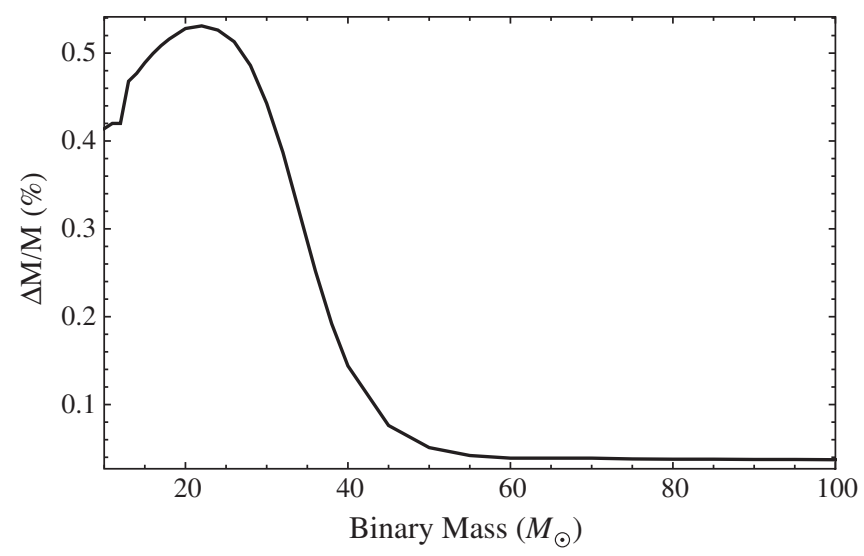

FIG. 11. Bias in mass estimation for the $\chi=0.5$ case, based on a comparison of $\mathrm{T} 1$ and $\mathrm{T} 4$ hybrids.

Advanced detectors, they can still be used to estimate the parameters of a binary, and the question remains what the accuracy of the parameter estimation can in principle be, and whether this accuracy is sufficient for GW astronomy applications in the near future.

To give an indication of what these errors might be, we can calculate the mass bias from our mismatch calculations-by which we mean the error in our estimate of the binary's mass, if we were to assume that it was given by the model waveform that exhibits the lowest mismatch with the true waveform. For the least accurate case we have considered here, $\chi=0.5$, with a hybrid matching frequency of $M \omega_{m}=0.05$, the worst mass bias is $0.54 \%$ for $M=$ $22 M_{\odot}$. The results for masses up to $100 M_{\odot}$ are shown in Fig. 11. We note for comparison that the results in Ref. [23] suggest that the PN waveforms that can be used for searches for binaries with $M<10 M_{\odot}$ have relatively high mass biases, around $20 \%$ for $M=10 M_{\odot}$. In addition, the results in Ref. [49] suggest that the mass bias from using 3.5PN TaylorT1 inspiral templates to detect phenomenological waveforms is around 10\%. These results of course also suggest that hybrids that are accurate enough for parameter estimation are also needed for binaries below $10 M_{\odot}$.

We emphasize that the true bias in the mass, when optimizing over all of the intrinsic and extrinsic parameters, may differ from that indicated in Fig. 11. While these results suggest that mass estimation errors will be very small with these waveforms, we defer a detailed analysis of parameter-estimation errors to future work.

\section{CONCLUSIONS}

In constructing hybrid PN + NR black-hole-binary waveforms there are three evident sources of error: the error in the numerical waveforms, the error in the PN waveforms, and the error introduced in the hybridization process. The error measure relevant for GW detection is the mismatch error, and in the context of current GW searches this error is required to be less than $3 \%$. 
We know from previous work $[22,29]$ that the mismatch error in the numerical waveforms is within this requirement by several orders of magnitude. We have shown in this paper that the mismatch error due to the hybridization process is also very low, at $\sim 0.03 \%$. The mismatch error due to the PN contribution to the hybrids, however, can be much larger, and dominates the error budget in all hybrids that can be produced with current PN and NR results.

Motivated by these observations, we have attempted to address the question of how many NR cycles (i.e., how few PN cycles) must be used in a hybrid in order for the hybrid to be sufficiently accurate for GW detection purposes. Our approach, which we justify in detail in Sec. VI, is to construct TaylorT4 + NR hybrids, and to treat these as the true GW signal $\left(\mathrm{NR}_{\mathrm{L}}\right)$, and then to calculate the mismatch between these and hybrids of Taylor $1+\mathrm{NR}_{\mathrm{L}}$. The mismatch is optimized with respect to the total mass of the binary, which we argue in Sec. VII is close to the mismatch resulting from a calculation of the fitting factor, which is the relevant quantity for GW detection. This allows us to make the most conclusive statements possible with currently available numerical simulations.

In the equal-mass nonspinning case, we find that very few NR cycles are necessary; five cycles (two orbits) are sufficient to be within the $3 \%$ mismatch requirement. Phase comparisons between PN and NR results suggest that the relative accuracy between TaylorT1 and TaylorT4 does not change significantly as the mass ratio is increased to $q=4$ [22], so we might expect that the length requirements are roughly the same for larger mass ratios. On the contrary, we find that the length requirements increase with the mass ratio, and for $q=415$ cycles (seven orbits) are necessary. We conclude that (1) we cannot infer NRwaveform length requirements directly from PN-NR phase comparisons, and (2) at higher mass ratios $q>4$ we will require yet longer NR waveforms, but more extensive studies will be required to determine how many.

Because of the greater PN errors for spinning binaries, more cycles are generally needed than in the nonspinning case. Our results are summarized in Table II. We have considered only equal-mass spinning cases, but we can conclude from these results that for unequal-mass spinning binaries, more NR cycles will be needed than in any of the cases we have considered here.

A conservative summary of our results would be that in the cases we have considered, simulations of ten orbits before merger should be sufficient to produce hybrids that are accurate enough for GW detection purposes. The resulting hybrids will be indistinguishable for SNRs in Advanced detectors of less than 30 for masses above $20-30 M_{\odot}$. The important caveat to our results is that they apply only to the "cheapest" cases. Nonspinning binaries with $q>4$ and unequal-mass spinning binaries will require more NR cycles, and we have not considered any cases with precessing spins.
These conclusions depend strongly on the current state of the art. The advent of more accurate PN results (for example higher-order spin contributions) could reduce these length requirements, as might a more robust quantification of the errors in PN methods. We have also not considered EOB results, for which the errors may be far lower, with a corresponding drop in the NR-waveform length requirements.

In addition to being relevant to the construction of hybrids for analytic waveform models, our results also have direct bearing on two current efforts in the NR, data analysis and analytical modeling communities.

In the second stage of the NINJA project [16] hybrid waveforms are being injected into detector noise to test search pipelines. The hybrids are constructed with matching frequencies in the range we have considered, but in most cases the matching will be around $M \omega=0.07$. In general, our results suggest that these hybrids will have a mismatch error of greater than $3 \%$ for masses lower than $20-30 M_{\odot}$.

The complementary NR-AR project [16] represents a community-wide effort to produce a large number of NR waveforms to calibrate analytic models. The nominal requirement for these waveforms is that they include $20 \mathrm{GW}$ cycles (ten orbits) before merger. From the point of view of constructing hybrid waveforms for GW detection with typical PN approximants, our results suggest that these waveforms will be long enough along the branches of the parameter space that we have considered, but possibly not for high-mass-ratio cases, some unequal-mass spinning cases, and perhaps also cases with precession.

We have presented a general method to assess NRwaveform length requirements, but our study only begins to address this question. Further work is needed to develop an easy-to-implement and robust method to estimate these requirements for configurations that have not yet been simulated in NR codes; work in this area is already underway [56]. It would be useful to make estimates of waveform length requirements for the construction of analytic models, both phenomenological and EOB; to extend this study to possible future detectors, like LISA [57] and the Einstein Telescope [58]; and, finally, much more work is required to understand the length requirements for parameter estimation, and to balance the needs of GW astronomy with the computational cost of numerical simulations.

\section{ACKNOWLEDGMENTS}

We thank Steve Fairhurst and Harald Pfeiffer for useful discussions, and Sukanta Bose and Doreen Müller for helpful comments on the manuscript. M. Hannam was supported by an FWF Lise-Meitner Fellowship (M1178N16) and a Science and Technology Facilities Council Advanced Fellowship (ST/H008438/1). S. Husa was supported by Grant No. FPA-2007-60220 from the Spanish Ministry of Science, the Spanish MICINNs ConsoliderIngenio 2010 Programme under grant MultiDark 
CSD2009-00064, and DAAD Grant No. D/07/13385. F. Ohme thanks the IMPRS for Gravitational Wave Astronomy and the DLR (Deutsches Zentrum für Luftund Raumfahrt) for support. P. A. was supported in part by NSF Grants No. PHY-0653653 and No. PHY-0601459, and the David and Barbara Groce Fund at Caltech. BAM simulations were carried out at LRZ Munich, ICHEC Dublin, the Vienna Scientific Cluster (VSC), at MareNostrum at Barcelona Supercomputing CenterCentro Nacional de Supercomputación (Spanish National Supercomputing Center), and CESGA, Santiago the Compostela.
[1] P. Ajith et al., Classical Quantum Gravity 24, S689 (2007).

[2] P. Ajith et al., Phys. Rev. D 77, 104017 (2008).

[3] P. Ajith, Classical Quantum Gravity 25, 114033 (2008).

[4] P. Ajith et al., arXiv:0909.2867.

[5] L. Santamaría et al., Phys. Rev. D 82, 064016 (2010).

[6] R. Sturani et al., J. Phys. Conf. Ser. 243, 012007 (2010).

[7] A. Buonanno et al., Phys. Rev. D 76, 104049 (2007).

[8] T. Damour and A. Nagar, Phys. Rev. D 77, 024043 (2008).

[9] T. Damour, A. Nagar, E. N. Dorband, D. Pollney, and L. Rezzolla, Phys. Rev. D 77, 084017 (2008).

[10] T. Damour, A. Nagar, M. Hannam, S. Husa, and B. Brügmann, Phys. Rev. D 78, 044039 (2008).

[11] J. G. Baker et al., Phys. Rev. D 78, 044046 (2008).

[12] A. H. Mroue, L. E. Kidder, and S. A. Teukolsky, Phys. Rev. D 78, 044004 (2008).

[13] T. Damour and A. Nagar, Phys. Rev. D 79, 081503 (2009).

[14] A. Buonanno et al., Phys. Rev. D 79, 124028 (2009).

[15] Y. Pan et al., Phys. Rev. D 81, 084041 (2010).

[16] http://www.ninja-project.org/.

[17] B. Aylott et al., Classical Quantum Gravity 26, 114008 (2009).

[18] B. Aylott et al., Classical Quantum Gravity 26, 165008 (2009).

[19] L. Blanchet, Living Rev. Relativity 9, 4 (2006), http:// www.livingreviews.org/lrr-2006-4.

[20] M. Hannam, Classical Quantum Gravity 26, 114001 (2009).

[21] M. Boyle et al., Phys. Rev. D 76, 124038 (2007).

[22] M. Hannam, S. Husa, F. Ohme, D. Müller, and B. Brügmann, Phys. Rev. D 82, 124008 (2010).

[23] A. Buonanno, B. Iyer, E. Ochsner, Y. Pan, and B. S. Sathyaprakash, Phys. Rev. D 80, 084043 (2009).

[24] Advanced ligo, http://www.ligo.caltech.edu/advLIGO.

[25] B. P. Abbott et al. (LIGO Scientific), Phys. Rev. D 80, 047101 (2009).

[26] B. P. Abbott et al. (LIGO Scientific), Phys. Rev. D 79, 122001 (2009).

[27] L. Lindblom, B. J. Owen, and D. A. Brown, Phys. Rev. D 78, 124020 (2008).

[28] C. Cutler and E.E. Flanagan, Phys. Rev. D 49, 2658 (1994).

[29] M. Hannam et al., Phys. Rev. D 79, 084025 (2009).

[30] D. Pollney, C. Reisswig, N. Dorband, E. Schnetter, and P. Diener, Phys. Rev. D 80, 121502 (2009).

[31] D. Pollney, C. Reisswig, E. Schnetter, N. Dorband, and P. Diener, arXiv:0910.3803.

[32] S. T. McWilliams, B. J. Kelly, and J. G. Baker, Phys. Rev. D 82, 024014 (2010).
[33] J. Abadie et al. (LIGO Scientific), Classical Quantum Gravity 27, 173001 (2010).

[34] B. Brügmann et al., Phys. Rev. D 77, 024027 (2008).

[35] S. Husa, J. A. González, M. Hannam, B. Brügmann, and U. Sperhake, Classical Quantum Gravity 25, 105006 (2008).

[36] M. Hannam, S. Husa, U. Sperhake, B. Brügmann, and J. A. González, Phys. Rev. D 77, 044020 (2008).

[37] M. Hannam, S. Husa, B. Brügmann, and A. Gopakumar, Phys. Rev. D 78, 104007 (2008).

[38] Y. Pan et al., Phys. Rev. D 77, 024014 (2008).

[39] M. Boyle, D. A. Brown, and L. Pekowsky, Classical Quantum Gravity 26, 114006 (2009).

[40] L. Blanchet, G. Faye, B. R. Iyer, and S. Sinha, Classical Quantum Gravity 25, 165003 (2008).

[41] K. G. Arun, A. Buonanno, G. Faye, and E. Ochsner, Phys. Rev. D 79, 104023 (2009).

[42] A. Buonanno, G. B. Cook, and F. Pretorius, Phys. Rev. D 75, 124018 (2007).

[43] J. G. Baker, J.R. van Meter, S.T. McWilliams, J. Centrella, and B.J. Kelly, Phys. Rev. Lett. 99, 181101 (2007).

[44] A. Gopakumar, M. Hannam, S. Husa, and B. Brügmann, Phys. Rev. D 78, 064026 (2008).

[45] I. Hinder, F. Herrmann, P. Laguna, and D. Shoemaker, Phys. Rev. D 82, 024033 (2010).

[46] M. Campanelli, C. O. Lousto, H. Nakano, and Y. Zlochower, Phys. Rev. D 79, 084010 (2009).

[47] M. A. Scheel et al., Phys. Rev. D 79, 024003 (2009).

[48] Waveforms are available at http://www.black-holes.org/ Waveforms.html.

[49] S. Bose, S. Ghosh, and P. Ajith, Classical Quantum Gravity 27, 114001 (2010).

[50] A. Buonanno and T. Damour, Phys. Rev. D 59, 084006 (1999).

[51] A. Buonanno and T. Damour, Phys. Rev. D 62, 064015 (2000).

[52] T. Damour, P. Jaranowski, and G. Schäfer, Phys. Rev. D 62, 084011 (2000).

[53] T. Damour, Phys. Rev. D 64, 124013 (2001).

[54] A. Buonanno, Y. Chen, and T. Damour, Phys. Rev. D 74, 104005 (2006).

[55] M. Boyle et al., Phys. Rev. D 78, 104020 (2008).

[56] F. Ohme, M. Hannam, and S. Husa (unpublished).

[57] D. A. Shaddock, Pub. Astron. Soc. Aust. 26, 128 (2009).

[58] M. Punturo et al., Classical Quantum Gravity 27, 084007 (2010). 